\title{
Building Artificial Intelligence for Dermatological Practice
}

\author{
Brian Tian \\ IDOC Medical Holdings, Singapore \\ Email: briananthonytian@gmail.com
}

How to cite this paper: Tian, B. (2018) Building Artificial Intelligence for Dermatological Practice. Open Access Library Journal, 5: e4541.

https://doi.org/10.4236/oalib.1104541

Received: March 23, 2018

Accepted: April 16, 2018

Published: April 19, 2018

Copyright $\odot 2018$ by author and Open Access Library Inc.

This work is licensed under the Creative Commons Attribution International License (CC BY 4.0).

http://creativecommons.org/licenses/by/4.0/ (c) (i) Open Access

\begin{abstract}
Purpose: Wrinkles are the most visually obvious features of aging and are the prime target of a vast number of products (both medical and cosmetic). It is important for clinicians to be able to grade wrinkles objectively. Although wrinkles are easily recognisable for humans, it remains a very challenging task for computer vision systems to detect them automatically. In our center, we developed a wrinkle detection algorithm based on a technique called "reversible jump Markov chain Monte Carlo framework with delayed rejection". This system is able to accurately and rapidly detect wrinkles. Methods: 300 images were submitted to the analyser for reading. Each image was analysed with a million iterations in ten minutes. The same 300 images were sent to a dermatologist for post-analyser evaluation. The system was trained to detect major and minor wrinkles. The results were benchmarked against the reviewing dermatologist. Results: Out of 300 patients, the pickup rate for major wrinkles was $100 \%$. However, on average it would be able to trace out only approximately $56.5 \%$ of the entire length of the wrinkle. The analyser was also able to detect minor wrinkles. However, the detection rate was only $13.4 \%$. Conclusion: Our system is able to accurately detect all major wrinkles. This enables physicians to track progress of antiwrinkling techniques such as Botox or surgical facelifts. Our system is also low cost as the wrinkle detection can be simply based off simple photographs.
\end{abstract}

\section{Subject Areas}

Dermatology

\section{Keywords}

Aesthetics, Telemedicine, Teledermatology 


\section{Introduction}

Wrinkles are the most visually obvious features of aging and are the prime target of a vast number of products (both medical and cosmetic). It is important for clinicians to be able to grade wrinkles objectively. This would enable clinicians to discern genuine effects of any anti-wrinkling agents. However, even with photographs, it remains difficult to truly and comprehensively grade wrinkles. This is because humans tend to rely on gross scoring scales, such as the Glogau photoaging scale. It is tedious and impractical to be able to highlight each and every wrinkle, especially those that are shallow or small, and judge it objectively.

However, if there was a computer system that could detect wrinkles for us in a reproducible fashion, then perhaps wrinkle detection could be fast, accurate and very detailed. Such should a system exist, wrinkle detection could be further applied to age estimation, forensic application and many others [1].

Although wrinkles are easily recognisable for humans, it remains a very challenging task for computer vision systems to detect them automatically. Wrinkles can be of many various shapes, lengths and depths. Their appearances can also vary according to ethnicity, gender and age. Wrinkle appearances are also highly dependent on the acquisition environment (type of imaging resolution, presence of light and direction).

In our center, we developed a wrinkle detection algorithm based on a technique called "reversible jump Markov chain Monte Carlo framework with delayed rejection" [2] [3]. This system is able to accurately and rapidly detect wrinkles. The utilisation of such artificial intelligence represents the tip of an iceberg, and a change in practice paradigms that will come in due time.

\section{Methods}

This study was conducted in Singapore. This study was conducted in accordance according to the principles of Helsinki. 356 patients were recruited. Inclusion criteria: Chinese patients, no skin conditions. Under fixed settings, photographs were taken using an Apple iPhone 6 (Apple Inc., California, USA). The settings were: $20 \mathrm{~cm}$ from the face with natural white light coming from behind them.

The images taken were screened by a dermatologist. 56 images were rejected. 32 were rejected because the lighting was not acceptable, and created a gradient of contrast across the face. 24 images were rejected because various parts of the skin appeared to be eczematous.

The remaining 300 images were submitted to the analyser for reading. Each image was analysed with a million iterations in ten minutes. The same $300 \mathrm{im}$ ages were sent to a dermatologist for post-analyser evaluation.

The evaluations done by the single reviewing dermatologist, adhered to the following definitions and principles. We defined major wrinkles as deep lines that are clinically obvious and are typically those targeted for therapy by Botox (for example the typical creases made by contraction of the frontalis muscles, crows' feet, etc). Minor wrinkles were defined as any other skin creases that 
would not be clinically significant (for example a fine crease anywhere on the forehead that is not amenable to Botox injections).

The analysed images were judged on the following criteria:

1) The detection rate of major wrinkles and minor wrinkles. This detection rate was calculated using the dermatologist's calculation as the reference standard. Example, if the dermatologist detected 10 major and minor wrinkles, and the computer also detected 10 , then the detection rate was $100 \%$.

2) For wrinkles that were detected, the dermatologist would determine the actual length of that wrinkle and determine how much of that particular wrinkle was traced or highlighted by the analyser. For example, if that particular wrinkle was $7 \mathrm{~cm}$ long, and the detector only traced it for $4 \mathrm{~cm}$, then it would have highlighted only $57.1 \%$ of that wrinkle.

\section{Results}

Of the 356 patients recruited initially, 84 were male, 272 were female. Of the 56 rejected patients, 32 were male and 24 were female. The final data set of $300 \mathrm{pa}-$ tients thus included 52 males and 248 females. The average age (and range) of the 300 patients was $52.3(27-72)$ years old.

The analyser was able to detect the presence of all major wrinkles, however it would not be able to trace out their full lengths. Out of 300 patients, the pickup rate for major wrinkles was $100 \%$. However, on average it would be able to trace out only approximately $56.5 \%$ of the entire length of the wrinkle.

The analyser was able to detect minor wrinkles. However, the detection rate was only $13.4 \%$. Erroneous detections occurred as well. There were no cases of erroneous detections for major wrinkles. This meant that there was no instance where the detector detected what seemed like a major wrinkle, where in fact there was none. However for minor wrinkles, the false detection rate was $22.5 \%$. This occurred because the analyser could not differentiate straight line segments well-it would detect fine facial hairs as wrinkles, or lighting artefacts as wrinkles as well.

Figure 1 demonstrates the image at the start of the analysis process. Figure 2 shows the same image at the end of the analysis process. Figure 3 shows the platform that this algorithm was running on.

\section{Discussion}

Computer vision is an area of research that is gaining much focus and traction. The ability to train a computer to identify and recognise anatomical structures, is the first step to developing systems that can make complex medical decisions. Thereafter, only then can computer systems deliver therapy, such as dispensing medicines or performing robotic surgery.

Localization and detection of wrinkles is a challenging problem. The reversible jump Markov chain Monte Carlo is one of the many techniques used to perform wrinkle detection. Kwon and Lobo [4] used active contour models to 


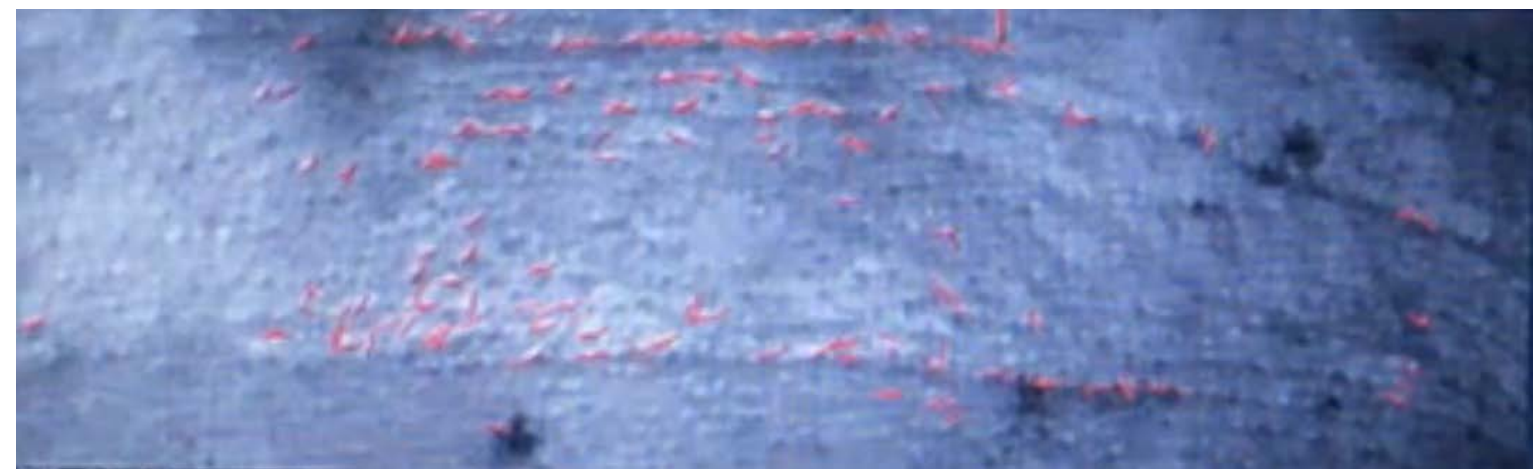

Figure 1. At the beginning.

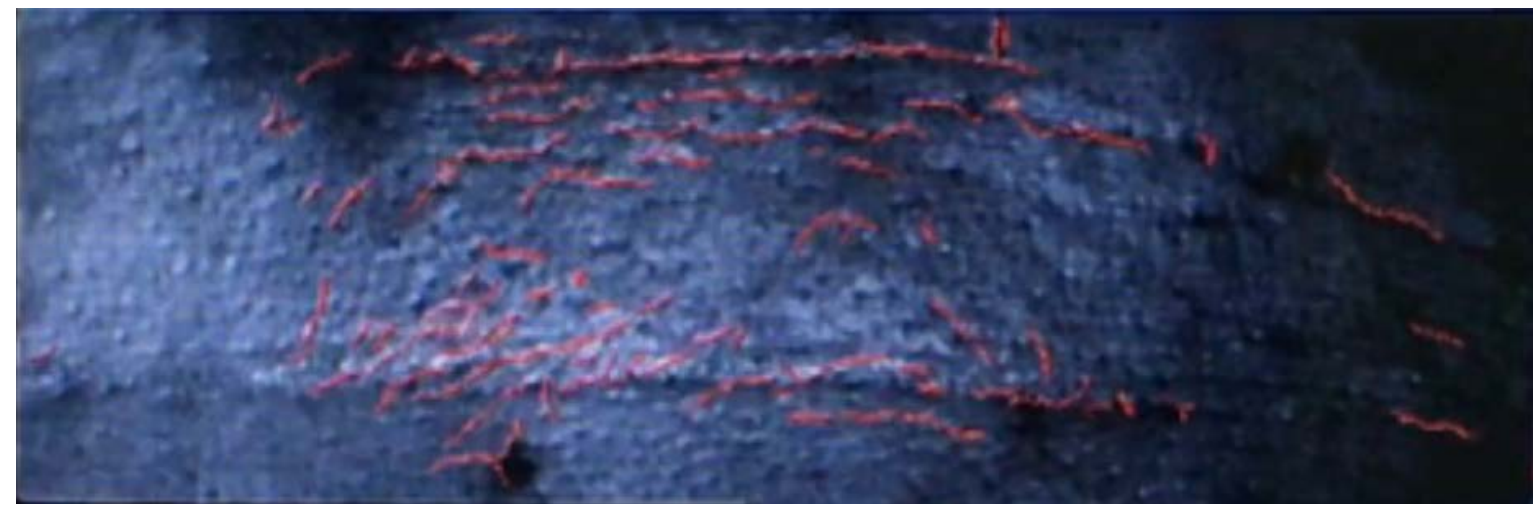

Figure 2. At the end.

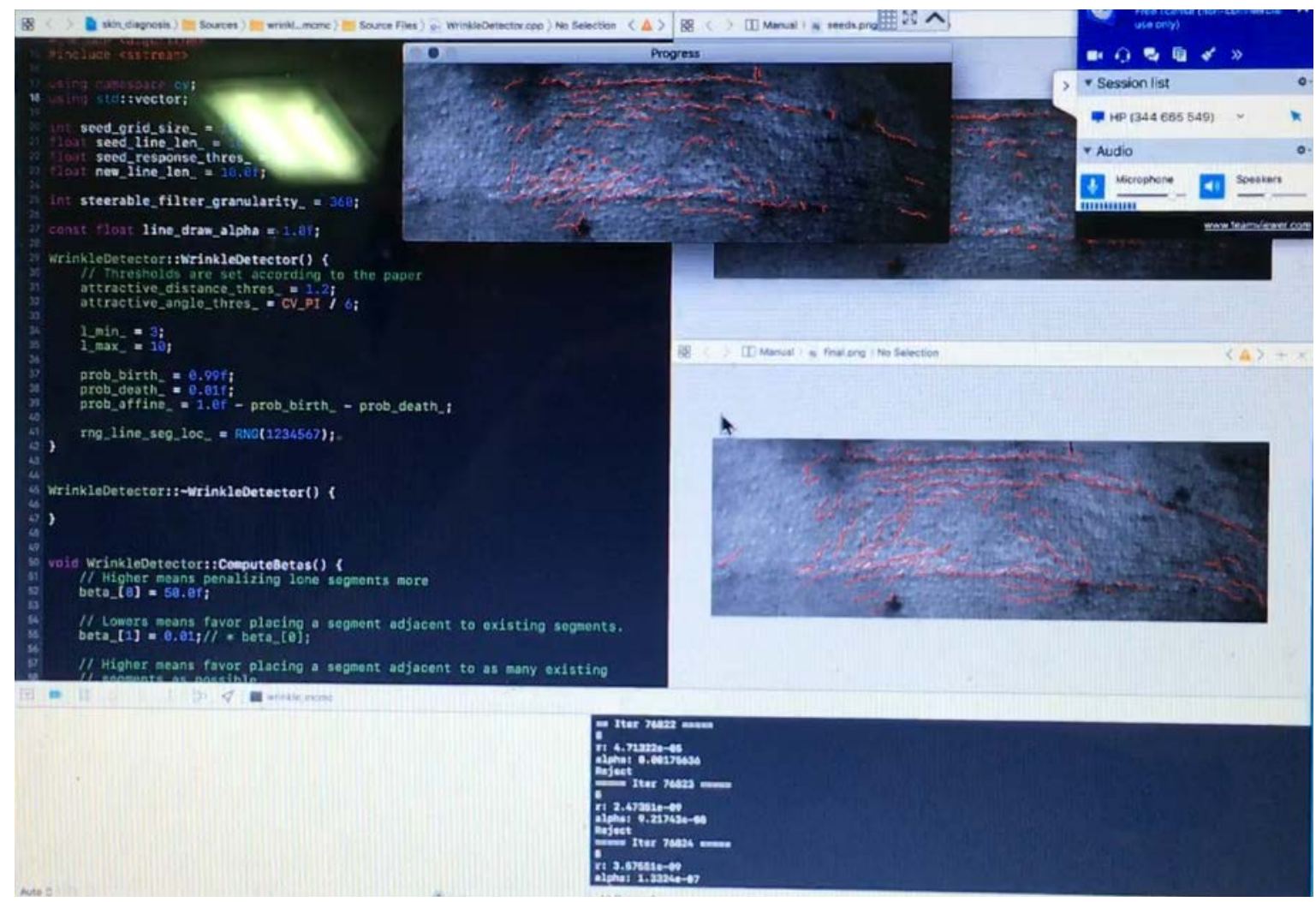

Figure 3. The algorithm. 
localize wrinkles in high resolution images. Cula et al. [5] reported work on localization and assessment of severity of facial wrinkles. The approach is based on enhancement of intensity gradients due to wrinkles using Gabor filters and subsequent thresholding of filter responses. Soitca et al. [6] analysed wrinkles as edges. Batool and Chellappa [7] proposed a stochastic wrinkle detection method based on the marked point process.

Our system is able to accurately detect all major wrinkles. This enables physicians to track progress of antiwrinkling techniques such as Botox or surgical facelifts. Our system is also low cost as the wrinkle detection can be simply based off simple iPhone photographs. When compared to a dermatologist, the system performs equally well in detecting major wrinkles, without the hassle and time needed to analyse these photographs. On average, the dermatologist took 30 minutes to an hour to evaluate a single photograph. Conversely, the system took only 10 minutes to do.

A few areas of improvements are however necessary. Firstly, there is a requirement for certain fixed settings to standardise the photographs, prior to analysis. Work needs to be done to teach the algorithm to filter or neutralise the environmental effects. Secondly, the algorithm is not able to trace out and thus measure the wrinkle length and depth. Thirdly, the system cannot identify fine wrinkles as it is unable to differentiate them from facial hairs. Fourthly, there also remains a need to adopt this technique in identifying other skin features such as pores and pimples.

As this system develops and its capabilities expand, its utility in clinical practice will be inevitable. Eventually, this will be incorporated into robotic systems that can identify, diagnose and even operate on patients.

\section{References}

[1] Batool, N., Taheri, S. and Chellappa, R. (2013) Assessment of Facial Wrinkles as a Soft Biometrics. 2013 10th IEEE International Conference and Workshops on Automatic Face and Gesture Recognition (FG), Shanghai, 22-26 April 2013, 1-7. https://doi.org/10.1109/FG.2013.6553719

[2] Green, P.J. (1995) Reversible Jump Markov Chain Monte Carlo Computation and Bayesian Model Determination. Biometrika, 82, 771-732. https://doi.org/10.1093/biomet/82.4.711

[3] Green, P.J. and Mira, A. (2001) Delayed Rejection in Reversible Jump Metropolis-Hastings. Biometrika, 88, 1035-1053. https://doi.org/10.1093/biomet/88.4.1035

[4] Kwon, Y.H. and Lobo, da V. (1994) Age Classification from Facial Images. 1994 IEEE Computer Society Conference on Computer Vision and Pattern Recognition, Seattle, WA, 21-23 June 1994, 762-767.

[5] Cula, G.O., Bargo, P.R. and Kollias, N. (2009) Assessing Facial Wrinkles: Automatic Detection and Quantification. Proceedings of SPIE, 7161, 71610J-71610J-6. https://doi.org/10.1117/12.811608

[6] Stoica, R., Descombes, X. and Zerubia, J. (2004) A Gibbs Point Process for Road Extraction from Remotely Sensed Images. International Journal of Computer Vision, 57, 121-136. https://doi.org/10.1023/B:VISI.0000013086.45688.5d 
[7] Batool, N. and Chellappa, R. (2012) Modeling and Detection of Wrinkles in Aging Human Faces Using Marked Point Processes. 12 th European Conference on Computer Vision “What's in a Face?” Workshop, Florence, October 2012, 178-188. https://doi.org/10.1007/978-3-642-33868-7_18 